\title{
3D Reconstruction from Truncated Rotational Angiograms Using Linear Prediction
}

\author{
Ramesh R. Galigekere and David W. Holdsworth \\ Imaging Research Laboratories, Robarts Research Institute, \\ 100 Perth Drive, P.O. Box 5015, London, ON. N6A 5K8, CANADA \\ \{ramesh, dholdswo\}@imaging.robarts.ca
}

\begin{abstract}
For obtaining high-resolution reconstruction of the cerebral vasculature, cone-beam projections in 3D computed rotational angiography (CRA) are acquired over a circular field of view (FOV) of $28 \mathrm{~cm}$, resulting in a truncation of the data. This results in erroneous values of reconstruction within the region of interest that worsens laterally towards the periphery. In this paper, an application of linear prediction is explored for alleviating the effects of truncation in CRA, and its impact on image registration and also reprojection, an important tool in $3 \mathrm{D}$ visualization and image enhancement algorithms in CRA. New observations on the effects of taper in the extrapolated segment on filtered projections, and their implications on 3D reconstruction in CRA lead to windowed extrapolation. Results of the new algorithms on a mathematical phantom and real data are promising.
\end{abstract}

\section{Introduction}

Three-dimensional (3D) computed rotational angiography (CRA) using an Xray image intensifier based $\mathrm{C}$-arm imaging system is a relatively new modality useful in cerebral angiography and neuro-interventional procedures [123]. For obtaining high-resolution reconstruction of the cerebral vasculature, the conebeam projections in CRA are now acquired over a circular field of view (FOV) of radius $28 \mathrm{~cm}$., resulting in truncated projections of the object. Reconstruction in CRA is performed by the Feldkamp algorithm [4,5], a (weighted) convolutionbackprojection procedure. It is well known in $2 \mathrm{D}$ that data-truncation results in errors in reconstruction within the region of interest (ROI), which becomes stronger towards the periphery. In 3D, erroneous values within the ROI or artifacts in the peripheral parts affect image registration (intensity-based and others) and reprojection, compromising the accuracy of image-guidance in interventional procedures. Reprojection is an important tool in 3D visualization and image enhancement algorithms 6789. Truncation-artifacts can also impact the result (visibility of small vessels and the speed) of thresholded maximum intensity projection [8], thus affecting the efficacy of image registration. The situation in $3 \mathrm{D}$ is different from that in $2 \mathrm{D}$ wherein one can visualize the reconstruction well within the ROI and those in the peripheral regions by varying the window parameters. 
Truncation due to a circular FOV amounts to a (lateral) truncation of each of the rows of the $2 \mathrm{D}$ projection data, and correction procedures similar to those applied in the 2D case are applicable. The effects of truncation in CT, and several methods for alleviating the same - generally involving the fitting of a smooth function over the region of truncation (ROT) - have been previously studied [10 11 12 12 13 14 15 15 16 17 18 . Recently, windowed 'symmetric mirroring' 19 was reported to have been used in CRA 2. However, the overall procedure of Ref. [19] seems to be specific (e.g., the parameter values, and the assumption of the existence of two 'interior' data points with values twice those at the edge-points). This paper considers an application of linear prediction (LP) for extrapolating CRA data, initial results associated with which were reported in 7]. LP extrapolation is efficient, involves minimal assumptions on the nature of data, produces a smooth extension, and is flexible to tackle other types of incompleteness in CT [18. It is observed here that LP extrapolation tends to over-estimate the data, and windowed extrapolation is proposed for alleviating the problem. In this context, the effects of tapering the extended segment on the filtered projections within the region of support, is studied. No explicit work of this nature has been reported in the literature, although the requirement of 'smoothness' in extrapolation is well known. Our study complements existing knowledge and provides additional insight. The basic study is performed in 2D, and the resulting methods and observations are extended to perform $3 \mathrm{D}$ reconstruction from truncated projection-images.

\section{Methods}

\subsection{Computed Rotational Angiography (CRA)}

CRA is a subset of cone-beam CT involving the reconstruction of contrasted cerebral vasculature from rotational angiograms obtained from an X-ray image intensifier based C-arm imaging system. Reconstruction is performed by the Feldkamp method [4], an extension of the fan-beam convolution backprojection (CBP) algorithm to 3D [4523. A truncation of the 2D projection data due to a circular FOV in CRA amounts to a lateral truncation, and the problem is essentially that of a truncation of the rows of the $2 \mathrm{D}$ data. Since filtering kernels in CT are non-causal and of infinite support, data truncation results in incorrect values of the filtered signal even within the ROI. From the frequency-domain perspective, truncation amounts to a multiplication by a rectangular window, and hence to a convolution of the Fourier transform of the true data by the sinc function. Thus, it is primarily the filtering stage in which errors due to truncation are introduced.

\subsection{Extrapolation of Projections by Linear Prediction}

Let $f(x, y)$ represent the object, and $p_{\theta}(t)$ its projection at angle $\theta$. Reconstruction from $\mathrm{CBP}$ requires the data to be available over the entire projection 
domain i.e., $\left\{p_{\theta}(t), t \in[-b, b], \theta \in[0, \pi)\right\}$. Consider reconstructing the object from a set of projections available on the interval $\{[-a, a](a<b)\}$ (region of support, ROS). In the following, we consider an extension of the 1D projections in 2D CT in the t-variable, followed by a reconstruction from CBP. The resulting observations and their implications on 3D CRA are discussed subsequently.

Autoregressive Extrapolation (ARE): The design of efficient algorithms for analysis, processing and synthesis can often be done under the framework of a model. Such a model can be used in several signal processing tasks. A powerful model involves linear prediction (LP) [20], useful for extrapolation [17,18]. The autoregressive (AR) model is a special case of the LP model, described by the recursive difference equation:

$$
s(n)=-\sum_{k=1}^{M} a_{k} s(n-k)+e(n)=\tilde{s}(n)+e(n)
$$

where, $e(n)$ is the modeling error and $M$, the order of the model; $\tilde{s}(n)$ is an estimate of $s(n)$ from its past $M$ samples. Of the various algorithms for AR parameter estimation, Burg's algorithm 21] is efficient and also guarantees the stability of the filter coefficients. The purpose of extrapolation in CT is towards image reconstruction within an ROI by alleviating the effects of truncation, which can be achieved by a low-order prediction. ARE provides a (very) smooth transition to zero.

Signal extrapolation by AR modeling consists of two steps (1) modeling the data i.e, estimating the parameters $\left\{a_{k}, k=1,2, . ., M\right\}$ from the data segment, and (2) predicting the values (extrapolating) outside the ROS based on the following recursion:

$$
s(n)=-\sum_{k=1}^{M} a_{k} s(n-k), \quad i=n_{0}+1, n_{0}+2, \ldots .
$$

where $n_{0}$ is the extreme point of the ROS. Eq. (2) corresponds to the right-sided extension, and the extension beyond the left-side of the ROS is performed in a similar fashion.

Windowed ARE (WARE): ARE tends to over-estimate the data within the ROT and may produce extensions of inconsistent lengths. Due to the nature of the CT-kernel, over-estimation of the data results in a under-estimation of the filtered projection, and vice-versa. A reasonable alleviation methodology would be a windowing of the extensions smoothly to a point beyond the true ROT, to compensate for the possible under-estimation within the ROT:

$$
\begin{aligned}
s_{w e}(n) & =s_{e}(n) w_{R}(n), & & n=n_{0}+1, n_{0}+2, \ldots, n_{0}+N_{e} \\
& =s_{e}(n) w_{L}(n), & & n=-\left(n_{0}+1\right), \ldots,-\left(n_{0}+N_{e}\right)
\end{aligned}
$$


where $s_{e}$ is the extrapolated segment, $N_{e}$ length of the window, and L and $\mathrm{R}$ denote Left and Right, respectively. In the absence of prior knowledge to the contrary, a smooth and longer extension is preferable over a sharp transition.

\section{Results}

A mathematical phantom i.e., that due to Shepp and Logan modified to mimic a slice of neuro-angiography-volume was created (Fig. 1(a)). 180 projections of the phantom have been considered in the simulation experiments. The effects of truncation on image reconstruction are evident in Fig. 1(c). The truncated projections were extended by AR modeling, and windowed ARE with different extents of the cosine-window. Each of the projections in the resulting sinograms were filtered by the Shepp-Logan (SL) kernel. The normalized root-mean-square error (NRMSE) of the filtered versions of the truncated and extrapolated projections were computed (over the ROS) with respect to those of the complete projections. The values of NRMSE are plotted as a function of the window (taper) index $i_{w}$ in Fig. 2 (a); the zeroeth point corresponds to unwindowed ARE. The rest of the points $i_{w}$ indicate the extent of windowing, $N_{w}$, expressed by:

$$
N_{w}\left(i_{w}\right)=N_{h}\left[1-0.1\left(i_{w}-1\right)\right], \quad i_{w}=1,2, . ., 9
$$

where $N_{h}=N_{s} / 2\left(N_{s}=\right.$ length of the available signal-segment). Eq. प 4 indicates progressively tapered windowing in steps of $10 \%$ of $N_{h}$. Note the presence of a point/region of minimum/low error. Images were reconstructed from the various sinograms, by the CBP method with the SL-kernel. The NRMSE of the reconstructions with respect to the original phantom (over the ROI) are also plotted in Fig. 2. Notice the similarity in the behaviour of the error in reconstruction and that associated with the filtered-projections.

Based on the success of WARE, we introduce a windowed version of the following simple extrapolation (SE):

$$
\begin{aligned}
p_{\theta}(n) & =p_{\theta}\left(n_{0}\right), & n \in\left[n_{0}+1, n_{0}+N_{e}\right] \\
& =p_{\theta}\left(-n_{0}\right), \quad & n \in\left[-\left(n_{0}+1\right),-\left(n_{0}+N_{e}\right)\right]
\end{aligned}
$$

The prime motivation for considering the above method is its sheer efficiency.

Fig. 2(b) shows a plot of the NRMSE associated with SE and windowed SE (WSE) - both in the filtered-sinogram domain, and the respective reconstructions over the ROI. The NRMSE associated with the filtered projections with SE is seen to be higher than that associated with unwindowed ARE. Not surprizingly, (unwindowed) ARE is superior to SE. The error-curves associated with the windowed versions, however, are nearly identical - which is an interesting result.

Reconstructions from some of the extensions discussed above are shown in figs. 3 and 4 . The image (a) in each of the sets demonstrates the effects of very gradual, smooth transition well beyond the true support of the projections. Notice the regions in the periphery of (and beyond) the ROI to be very 'clear'. 
However, the values in the reconstructed image were slightly lower than the true values (i.e., under-estimated). This can be easily visualized by the plots of the values of the reconstructions (within the ROI) over a line running vertically through the central vessel and its two neighbors, in fig. 3(d) (this has an important implication in CRA: the extrapolations of each of the rows should be performed in a manner to maintain consistency in the amplitudes contributed to the reconstruction from the rows with different extents of truncation). The effect of windowing/tapering can be seen in the second image (b) in each of the sets - although the appearance of a circular band can be seen, the values in the ROI were very close to the true values, as also reflected by the lowest values of the NRMSE. The final image (c) in each set shows the undesirable effect of tapering the extension sharply (by the same 'smooth' window). Although one may be able to visualize the small objects in the peripheral region by adjusting the window and its level, such an artifact will adversely affect registration and also the quality of reprojections in 3D. The preceding results support the remarks made in the previous sections. Note that a smooth windowing of ARE beyond the true extent of the data is superior to unwindowed ARE. Thus, a smooth, gradual extension beyond the ROT seems 'safer' in general than steep tapering (even if it coincides with the true extent of the projection data). Finally, WARE is not significantly better than WSE.

Consider the results of an application of the preceding algorithms on human patient data acquired using our prototype C-arm system (Siemens Multistar; details of data acquisition are reported in Ref [7]). A sample projection (truncated due to circular FOV) at $-95.62^{0}$ (corrected for distortions [22 23]) is displayed in fig. 5(a). The 3D image was reconstructed from 130 projections by the Feldkamp algorithm using projection matrices in the backprojection-step. A ray-driven reprojection [7] of the volume at the same angle is shown in fig. 5(b). Note the effects of bowl-shaped artifact (intensifying laterally towards the edges, often in the form of circular arc/rings) in the volume-slices, and a reduced contrast and detail. Each of the rows in a cone-beam projection was then extended on either side by an AR model of order 5 . This process was repeated on all the projections. Improvement in the reconstruction from ARE-projections is clearly seen in the reprojection displayed in fig. 5(c). The effect of possibly over-estimated ARE within the ROT can also be seen in terms of reduced intensity on the upper and lower parts. The result of WSE displayed in 5(d) shows an improvement in this aspect (i.e, notice an improved uniformity in intensity). Windowing was performed from the point of truncation to the zero-padding-edge (convolution was performed through FFT). Note that computation can be avoided in WSE by the use of a table. Thus, this simple and inexpensive method of extrapolation can be quite effective in practice.

\section{Conclusion}

An application of autoregressive modeling for data extrapolation towards improved 3D reconstructions from truncated rotational angiograms has been de- 

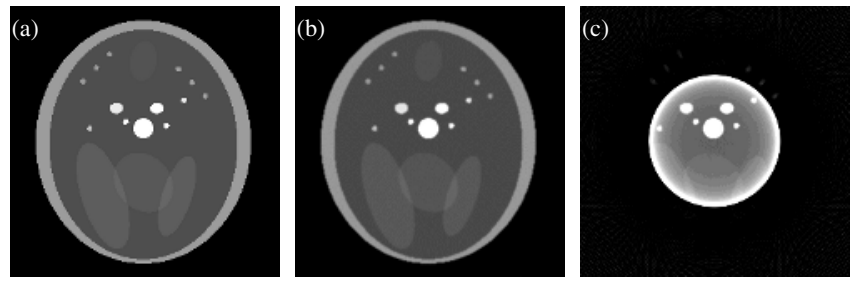

Fig. 1. (a) The phantom used in the experiments described in this paper. (b) Its reconstruction from CBP with the S-L filter from 180 complete projections. (c) Its reconstruction from projections truncated beyond the central 120 points
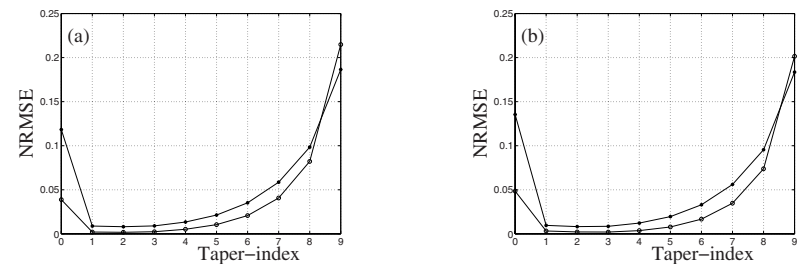

Fig. 2. NRMSE as a function of taper: (a) ARE, and (b) SE. In these plots, the zeroeth point corresponds to the unwindowed extrapolation; $i_{w}=1,2, \ldots, 9$ correspond to data tapered in steps of $10 \%$ of $N_{h}$ (expression 4). The NRMSE in the filtered sinogram is denoted by 'o' and that in the reconstruction by '*'
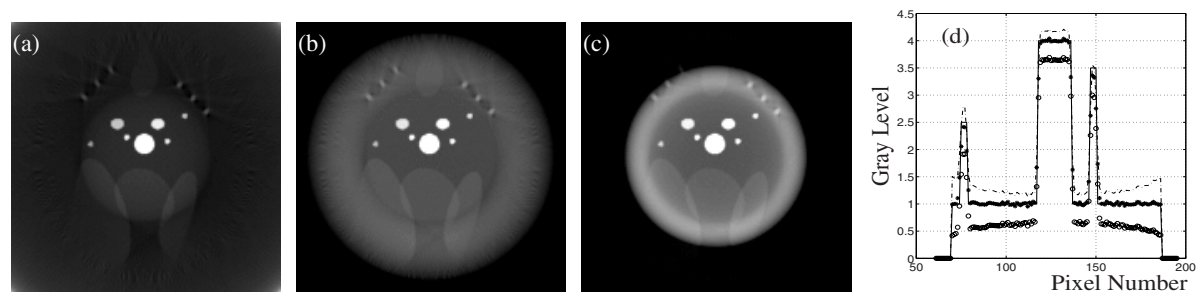

Fig. 3. Images reconstructed from (a) ARE-projections, and WARE-projections with $N_{e}$ corresponding to (b) the minimum error, and (c) $i_{w}=7$ in fig. 2. (d) Plots of reconstructions (within the ROI) over a line running vertically through the central vessel and its two neighbors. The solid line is the reference (original image); (o): ARE, $\left(^{*}\right)$ : WARE at $i_{w}=2$ (nearly coinciding with the solid line), and (-.): WARE at $i_{w}=7$
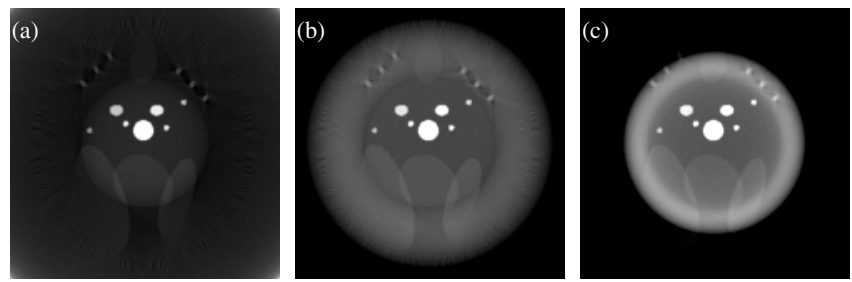

Fig. 4. Images reconstructed from (a) SE-projections, WSE-projections with $N_{w}$ corresponding to (b) the minimum reconstruction error (i.e., $i_{w}=2$ ) and (c) $i_{w}=7$ 

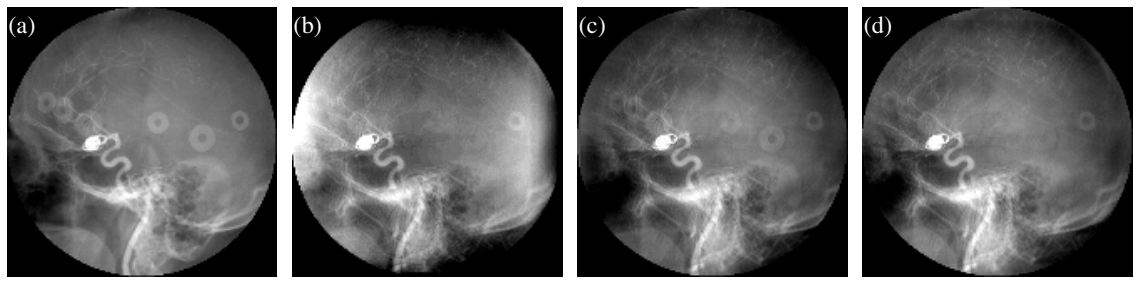

Fig. 5. (a) Original X-ray projection. Reprojection of the volume reconstructed from (b) truncated data due to circular FOV, (c) ARE-projections, and (d) WSE-projections

scribed. Filtering in the reconstruction algorithm being the primary step in which artifacts are introduced, the effect of truncation and taper associated with the extrapolations were studied. The study performed in 2D resulted in interesting observations. In particular, since ARE by itself may not result in data tapered to zero within an optimum extent, the idea of windowing AR-extended projection data was introduced. Gradual tapering resulted in under-estimated but relatively 'safe' reconstruction, whereas steep tapering can result in an undesirably strong bowl-shaped artifact. These observation are significant for 3D CRA because, information within the ROI as well as in a neighborhood of its periphery is useful. This is in view of applications such as registration and the use of reprojection for visualization and as a tool in image-enhancement algorithms. Further, windowed simple extrapolation involving no significant computation has been shown to work reasonably well. The proposed algorithms are flexible to be adapted to different scenarios in CT. Results of the new algorithms on simulated as well as real data show their efficacy. Future work involves optimizing the windowing/tapering strategy for best performance in CRA.

Acknowledgment. The financial assistance of the Canadian Institutes of Health Research (through grants GR-14973 and MOP-13356) is gratefully acknowledged. The second author is a Career Investigator supported by the Heart and Stroke Foundation of Ontario. Thanks are due to Mr. Todor Ivanov for help with visual examination of reprojections.

\section{References}

1. R. Fahrig, A.J. Fox, S. Lownie, and D.W. Holdsworth. Use of a C-arm system to generate true three-dimensional computed rotational angiograms: Preliminary in vitro and in vivo results. Am. J. Neuroradiol., 18:1507-1514, 1997.

2. K. Wiesent, K. Barth, N. Navab, P. Durlak, T. Brunner, O. Schuetz, and W. Seissler. Enhanced 3D-reconstruction algorithms for C-arm systems suitable for interventional procedures. IEEE Trans. Med. Imag., 19:391-403, 2000.

3. N. Navab, M. Mitschke, and O. Schuetz. Camera-augmented mobile C-arm (CAMC) applications: 3D reconstruction using a low-cost mobile C-arm. In Proc. MICCAI, pages 688-697, 1999. 
4. L.A. Feldkamp, L.C. Davis, and J.W. Kress. Practical cone-beam algorithm. J. Opt. Soc. Am. A, Opt. Image Sci., 1:612-619, 1984.

5. A.C. Kak and M. Slaney. Principles of Computerized Tomographic Imaging. IEEE Press, 1988.

6. R.R. Galigekere, K. Wiesent, and D.W. Holdsworth. On cone-beam Reprojection of a 3-D Reconstruction and its Applications. World Congress on Medical Physics and Biomedical Engineering (Med. Phy. 27 page 1396), Aug. 2000.

7. R.R. Galigekere, K. Wiesent, and D.W. Holdsworth. Cone-beam reprojection using projection matrices. IEEE Trans. Med. Imag. (to Appear), Oct. 2003.

8. R.R. Galigekere and D.W. Holdsworth. On Perspective Maximum Intensity Projection of CRA data. Proc. SPIE Symp. Medical Imaging, 4681:688-697, 2002.

9. R.R. Galigekere and D.W. Holdsworth. Cone-beam Maximum Intensity Projection Algorthims for Computed Rotational Angiography. To be submitted to Med. Phys.

10. R.M. Lewitt and R.H.T Bates. Image reconstruction from projections: I: General theoretical considerations. Optik, 50:19-33, 1978.

11. R.M. Lewitt and R.H.T Bates. Image reconstruction from projections: III: Projection completion methods (theory). Optik, 50:189-204, 1978.

12. R.M. Lewitt and R.H.T Bates. Image reconstruction from projections: IV: Projections completion methods (computational examples). Optik, 50:269-278, 1978.

13. J.C. Gore and S.Leeman. The reconstruction of objects from incomplete projections. Phys. Med. Biol, 25:129-136, 1980.

14. P.S. Tofts and J.C. Gore. Some sources of artefact in computed tomography. Phys. Med. Biol., 25:117-127, 1980.

15. G.T. Herman and R.M. Lewitt. Evaluation of a preprocessing algorithm for truncated CT projections. J. Comput. Assist. Tomogr., 5:127-135, 1981.

16. J.L. Lehr. Truncated-view artifacts: Clinical importance on CT. Am. J. Radiol., 141:183-191, 1983.

17. N. Srinivasa, V. Krishnan, K.R. Ramakrishnan, and K. Rajgopal. Image reconstruction from Truncated Projections: A Linear Prediction Approach. Int. Conf. Acoust. Speech Signal Process., Tokyo, pages 34.3.1-34.3.4, 1988.

18. K. Rajgopal, N. Srinivasa, and K.R. Ramakrishnan. Image reconstruction from incomplete projection data: A linear prediction approach. In C.T. Leondes, editor, Medical Imaging Systems, Techniques and Applications: Modalities, Gordon and Breach International Series in Engineering, Technology and Applied Science, 1997.

19. B. Ohnesorge, T. Flohr, K. Schwarz, J.P. Heiken, and K.T. Bae. Efficient correction for CT image artifacts caused by objects extending outside the scan field of view. Med. Phys., 27:39-46, 2000.

20. J. Makhoul. Linear prediction: A tutorial review. Proc. IEEE, 63:561-580, 1975.

21. S.J. Orfanidis. Optimum Signal Processing. McGraw-Hill International Edition, New Delhi, 1990.

22. R. Fahrig, M. Moreau, and D.W. Holdsworth. Three-dimensional computed tomographic reconstruction using a C-arm mounted XRII: Correction of image intensifier distortion. Med. Phys., 24:1097-1106, 1997.

23. R. Fahrig and D.W. Holdsworth. Three-dimensional computed tomographic reconstruction using a C-arm mounted XRII: Image-based correction of gantry motion nonidealities. Med. Phys., 27:30-38, 2000. 